Paine, Jun. T. F. \& Daniel, R. R. (1959). J. gen. Microbiol. 21, 203-204.

\title{
Attempts to Obtain Gram-Negative Rods from Staphylococci Treated with Penicillin
}

\author{
By T. F. PAINE, Jun. ANd R. R. DANIEL \\ Department of Microbiology, University of Alabama Medical Center, Birmingham, \\ Alabama, U.S.A.
}

SUMMARY: Gram-negative bacilli could not be obtained from the Oxford strain of Staphylococcus aureus nor from 15 other strains of S. aureus in the presence of benzylpenicillin.

In recent communications Crawford \& Abraham (1957) and Briggs, Crawford, Abraham \& Gladstone (1957) reported interesting morphological and physiological phenomena associated with the Oxford strain (NCTC 6571) and a penicillinase-producing strain (D3R) of Staphylococcus aureus. When the two strains were cultivated for less than $24 \mathrm{hr}$. in broth in the presence of penicillin, bacitracin or cephalosporin $\mathrm{C}$ and bacitracin, respectively, they were found to give rise to Gram-negative rods. Subcultures of the resulting Gram-negative rods differed from the original staphylococcal strains, not only in colonial morphology and biochemical reactions but in phage susceptibility and animal pathogenicity as well. Under certain cultural conditions it was reported possible to obtain re-emergence of the Gram-positive cocci from the Gram-negative rod cultures. The obvious implications of these apparently distinct reversible changes in morphological and physiological properties of staphylococci prompted us to undertake similar studies.

\section{METHODS}

In addition to the Oxford strain, 15 other strains of Staphylococcus aureus, mostly of recent human origin, were used in the present work. Each of the strains was plated out three consecutive times on brain heart infusion agar (Difco) to verify morphological and tinctorial homogeneity. The strains were all coagulase-positive and were completely inhibited by penicillin in concentrations of $0.038-0.076 \mu \mathrm{g} . / \mathrm{ml}$., as determined in a twofold tube dilution assay with inocula of 50-100,000 organisms.

One-tenth ml. of a c. $18 \mathrm{hr}$. broth culture of each strain was inoculated into $60 \mathrm{ml}$. brain heart infusion broth (Difco) contained in a screw-capped Erlenmeyer flask. After incubation for $2-3 \mathrm{hr}$, at $37^{\circ}$, when the cultures were in the logarithmic phase and the broth was slightly cloudy, sodium benzylpenicillin (1670 units/mg.; batch 29293, Glaxo Laboratories Ltd.) was added to give a final concentration of $1 \mu \mathrm{g} . / \mathrm{ml}$. With continued incubation the organisms lysed and the broth remained clear for periods ranging from 2 to 21 days, when the cultures again became cloudy. The flasks were opened, subcultures made to blood agar plates and smears made during the first $24 \mathrm{hr}$. of incubation and later when the cultures became cloudy. Aeration of duplicate cultures during incubation was effected by means of a rotary action flask shaker. 
The strains of Staphylococcus aureus which were used are listed below. The number of times each strain was cultured and examined for its ability to produce Gram-negative rods in the presence of penicillin is denoted by two numbers following the strain; whether the cultures were aerated or stagnant is indicated by an $\mathrm{A}$ or an $\mathrm{S}$ : Oxford strain 15 A, 6S; strain 209P (ATCC 6538P) 9A, 6S; strains (BB1), (DC4), (JC1), (LB2), (NL2), (399), (825), (852), (932), (944), (3406), (3559), (3565) and (3582) each $6 \mathrm{~A}, 6 \mathrm{~S}$.

\section{RESULTS}

Sixteen penicillin-sensitive strains of Staphylococcus aureus were cultured in the presence of benzylpenicillin for a total of 204 times (108 aerated, 96 stagnant); only Gram-positive cocci were found in the stained smears of the cultures and only staphylococcal colonies grew out on subculture to blood agar plates. The penicillin and broth controls included with each series of tests remained clear and showed no organisms on stained smear or on subculture.

Our inability to induce the emergence of Gram-negative rods in cultures of the Oxford and 15 other strains of staphylococci with penicillin is in sharp contrast to the observations of the Oxford workers. The reasons for this discrepancy in results, particularly with the Oxford strain, remain unknown. Though the Oxford workers interpreted the appearance of Gram-negative rods in their cultures as morphological and physiological variants of staphylococci, the possibility of an inadvertent introduction or the presence of a previously unrecognized contaminant is of foremost concern here and deserves comment. Inapparent and prolonged coexistence of two bacterial species is not an uncommon phenomenon; Barringer \& Dack (1935) documented such an arrangement with the bacteroides and several other micro-organisms. The Oxford workers attempted to eliminate the possibility that their staphylococcal strains were contaminated by picking single organisms by micro-manipulation for initiation of cultures. However, seeing individual small Gram-negative rods in the living unstained state might be difficult and it is conceivable that contaminants might not be detected on such a visual examination.

This work was aided by grant E-938(C2) from the National Institute of Allergy and Infectious Diseases, U.S. Public Health Service. One of us (R.R.D.) held a Lederle Medical Student Research Fellowship during the work.

\section{REFERENCES}

BARringer, S. J. \& DACK, G. M. (1935). Isolation of Bacterium typhosum when mixed with anaerobic, non-spore forming, Gram-negative rods (Bacteroides). Proc. Soc. exp. Biol., N.Y. 32, 1125.

Briggs, S., Crawford, K., Abraham, E. P. \& Gladstone, G. P. (1957). Some properties of Gram-negative bacilli obtained from a strain of Staphylococcus aureus in the presence of benzylpenicillin. J. gen. Microbiol. 16, 614.

Crawford, K. \& Abraham, E. P. (1957). The synergistic action of cephalosporin C and benzylpenicillin against a penicillinase-producing strain of Staphylococcus aureus. J. gen. Microbiol. 16, 604. 